\title{
La pertinence et de l'efficacité de régulations onusiennes en faveur de la démocratie en République Démocratique du Congo
}

\author{
Par FATU MATA Nadine*
}

\section{INTRODUCTION}

Cette étude essentiellement axée autour de «la pertinence et de l'efficacité de régulations Onusiennes en faveur de la démocratie en République Démocratique du Congo » entend répondre à la question du bien-fondé des interventions des nations Unies et de leur impact sur l'instauration de la démocratie dans ledit pays. En tant que telle, elle s'inscrit dans le cadre du séminaire des questions spéciales de l'histoire des idées politiques de la République Démocratique du Congo, prévu au programme de l'enseignement supérieur et universitaire au niveau du troisième cycle.

Sur le plan méthodologique, elle fait appel à la méthode systémique appuyée par la technique d'observation indirecte dans la mesure où, elle procède à l'examen du système onusien en compulsant le plus des documents possibles traitant de la matière relative à l'objet qui en constitue la toile de fond.

$\mathrm{Au}$ fond, il est question de jauger l'efficacité des régulations politiques onusiennes en faveur de la paix et de l'instauration de la démocratie dans ce pays, eu égard à la situation dans laquelle ce dernier se trouve plongé jusqu'aujourd'hui en cette matière.

En effet, nul n'ignore que, depuis son accession à l'indépendance, en date du 30 juin 1960, la République Démocratique du Congo est continuellement confrontée à des crises politiques récurrentes dont l'une des causes fondamentales se trouve être la contestation de la légitimité et de leurs animateurs. Cette contestation, lit-on dans l'exposé des motifs de la Constitution du 18 février 2006, a pris un relief particulier avec les guerres qui ont déchiré le pays de 1996 à 2003.

Par ailleurs, il faut noter qu'en dépit de ses immenses potentialités économiques, la République Démocratique du Congo connaît de nos jours une situation qui véhicule angoisse et ronge la trame du cœur sur le plan du développement et de l'application des principes démocratiques. Inscrite sur la liste des pays pauvres très endettés, classé parmi les Etats les plus corrompus du monde, elle tourne aujourd'hui l'une des pages les sombres de son histoire en raison notamment de l'instabilité politique et économique toujours permanente, aux conséquences désastreuses pour son développement et l'avenir de la démocratie devant un silence pusillanime de ses fils et filles intellectuels et non intellectuels.

Toutefois, devant cet état des choses, les Nations Unies ne sont pas restées indifférentes. Comme dans les années soixante, elles se sont mobilisées en vue de la promotion de

* Assistante à l'Institut Supérieur d'Etudes Sociales de Lubumbashi. 
la paix et de la stabilisation de la démocratie. Est-il nécessaire de préciser qu'on les a vues à cette fin présentes et actives dans les différentes négociations et multiples accords sur la République Démocratique du Congo, notamment à Lusaka, à Addis-Abeba et, finalement à Sun City où est né un nouvel ordre politique fondé sur une nouvelle Constitution, dite du 18 février 2006.

Sous son impulsion les élections qui ont permis à l'installation de nouvelles institutions politiques ont été organisées en 2006, bien que ne l'ayant pas été à tous les niveaux. En 2011, il a été organisé seulement les élections présidentielles et législatives au niveau national et ce, après une première modification de la Constitution. En effet, on est passé de deux tours à un seul tour. Il faut noter pour ces élections, organisées cette fois-ci sous l'égide de la CENI, les contestations ont été vives, discréditant ainsi l'image du pays et la raison d'être de la mission onusienne.

Alors que l'année 2016 venait de marquer la fin du mandat présidentiel et de l'Assemblée nationale aux côtés d'un sénat et des assemblées provinciales jamais renouvelés car toujours en place depuis 2006, aucune élection n'a été organisée pour marquer l'alternance politique au sommet de l'Etat. Cette situation honteuse, faut-il le souligner, n'est pas sans poser problème. Elle soulève la question de l'efficacité et de la pertinence des implications onusiennes en faveur de la Démocratie en République Démocratique du Congo et de la bonne foi de la classe politique au pouvoir dans ce pays..

Faut-il souligner que depuis cette prorogation anticonstitutionnelle des mandats des dirigeants politiques actuels, les relations entre sur l'Organisation des Nations Unies et la classe politique Congolaise au pouvoir ne sont pas aux beaux fixes. Cette dernière accuse sur l'Organisation des Nations Unies d'ingérence dans les affaires internes et de violer le principe de l'égalité souveraine des Etats.

Aussi, devant cet état des choses, nous a-t-il paru impérieux d'axer notre réflexion dans le cadre de cette étude, autour des Nations Unies et de la démocratie en République Démocratique du Congo. Pour ce faire, nous allons articuler l'essentiel de notre réflexions en trois points successifs ponctués par une brève conclusion; à savoir : les généralités sur l'Organisation des Nations Unies, les régulations politiques Onusiennes en République Démocratique du Congo, leur pertinence et efficacité en République Démocratique du Congo.

\section{A. GENERALITES SUR L'ORGANISATION DES NATIONS UNIES (ONU)}

Notons d'entrée de jeu que, l'Organisation des Nations Unies apparaît, de la lecture attentive de sa charte, comme le résultat inéluctable du déséquilibre socio-économique survenu à la suite d'innombrables problèmes posés, notamment par les guerres ou même antérieures à celles-ci. Créée sur les décombres de la Société des Nations (SDN), elle est dotée des organes assez bien définis dans leur structure comme dans leurs compétences. 
Plusieurs buts et finalités lui sont assignés qui cependant, se ramènent au désir de sauvegarder la paix et la sécurité internationales en vue de favoriser le progrès social et d'instaurer des meilleures conditions de vie ${ }^{1}$.

Aussi, dans le premier point, allons-nous focaliser l'essentiel de notre réflexion autour de la genèse des Nations Unies, de ses buts et principes ainsi que de ses structures organiques.

\section{GENESE DES NATIONS UNIES}

Une lecture attentive de la Charte des Nations Unies révèle que, la création de cette dernière apparaît comme une réaction aux causes de l'échec de la société des nations.

Sa constitution est, en fait, passée par plusieurs phases allant des travaux préparatoires à la conférence de San Francisco en passant par la déclaration de Washington, les conférences de Moscou et de Téhéran, les propositions du Dumbarton Oaks et la conférence de Yalta.

Est-il nécessaire de préciser que, la SDN, première Organisation Internationale à vocation universelle, avait failli à sa mission. Elle s'était en effet, révélée incapable de garantir la paix et la sécurité dans le monde. Elle n'avait pas pu arrêter la guerre de 1914, c'est ainsi qu'est né au cours des années le projet de créer une nouvelle Organisation Internationale fondée sur le principe d'une égalité de souveraineté de tous les Etats pacifiques. Cette organisation, c'est l'Organisation des Nations Unies dont la charte a été adoptée le 26 juin 1945 et entrée en vigueur le 24 octobre $1945^{2}$. A cet égard, lors de son voyage en Europe après les hostilités, le Président WOODROW Wilson déclarait : "Le fait de gagner la guerre seulement ne suffit pas. Il faut la gagner de façon à assurer la paix future du monde. Les peuples du monde doivent travailler ensemble pour éviter les guerres, trouver tes solutions en dehors des champs de batailles. Les hommes doivent éviter que les horreurs de 1914 ne puissent plus arriver. ${ }^{3}$

Quels sont donc les objectifs et les principes des Nations Unies?

\section{OBJECTIFS ET PRINCIPES DES NATIONS UNIES}

II faut noter de prime abord qu'en ce qui concerne les buts des Nations Unies, ceux-ci se trouvent définis dans le préambule ainsi que dans le chapitre premier de la Charte. En effet, cette dernière distingue quatre buts principaux, à savoir :

a) préserver les générations futures du fléau de la guerre qui « deux fois en l'espace d'une vie humaine », a infligé à l'humanité d'indicible souffrance.

1 Voir le préambule de la Charte des Nations Unies.

2 ROUGET, D., Le guide de la protection internationale des droits de 'homme, Dalloz, Paris, 2000, p.225.

3 KADORY, N.K., Organisation Internationale, éd. Essai, Lubumbashi, 2013, P, 23. 
En ceci, faut-il le souligner, le maintien de la paix (article $1^{\text {er }}$ ) et de la sécurité internationale constitue donc la raison d'être de la création de l'Organisation des Nations Unies ainsi que dans la charte de la SDN.

b) Réaliser la coopération internationale en résolvant les problèmes internationaux d'ordre économique, social, intellectuel et humanitaire, en développant et en encourageant le respect des droits de l'homme et les libertés fondamentales pour tous... (article 132).

c) La promotion et le respect des Droits de l'homme pour tous les peuples.

d) Développer entre les nations les relations amicales fondées sur le respect du principe de l'égalité des droits de peuples et de leurs droits à disposer d'eux-mêmes.

En vue de la réalisation de ses objectifs, soulignons que les Nations Unies et ses membres ont le devoir de conformer leurs actions aux principes ci-après :

a) L'égalité souveraine de tous les Etats;

b) Le non recours à la force et le règlement pacifique des différends (Article 28,3);

c) Le principe de bonne foi (Article 28,2);

d) La clause de la compétence nationale ou principe de non-ingérence (article 28,7).

\section{LES ORGANES DES NATIONS UNIES}

Les Nations Unies disposent de six organes principaux notamment :

\section{L'Assemblée Générale}

La composition de l'Assemblée Générale est organisée à l'article 9 de la Charte. Tous les Etats membres de l'Organisation des Nations Unies y sont représentés.

Un hommage rituel y est ainsi rendu à la souveraineté et à l'égalité des Etats : « ouverte à tous les membres, l'Assemblée incarne, selon l'expression empruntée à Charles ZORGBIDE, la démocratie à l'échelle internationale. " ${ }^{4}$

Signalons que l'Assemblée Générale dispose de compétences très larges, mais n'émet pas des décisions contraignantes pour les Etats.

\section{Le Conseil de Sécurité}

Composé de 11 (onze) membres dont cinq permanents et six non permanents avant l'amendement de l'article 23 de la Charte, le Conseil de Sécurité comprend depuis cet amendement le 17 décembre 1964, entré en vigueur le 31 août 1965 et, mis en application le 1er août 1966, quinze membres dont cinq permanents et dix non permanents.

Il faut préciser que les cinq membres permanents sont de grandes puissances bénéficiant dans les votes d'un droit de veto grâce auquel chacune d'elles à elle seule peut faire échouer tout projet de résolution qui ne rencontre pas son assentiment, quelle que soit par

4 ZORGBEDE, CH., Les Organisations Internationales, P.U.F., Paris, éd. Que sais-je? 1986, p25. 
ailleurs, la majorité des autres membres du Conseil qui l'aurait accepté. En ordre général, le maintien de la paix et de la sécurité internationale demeure la fonction primordiale du Conseil de sécurité (article 24 de la Charte). A ce titre, il est appelé à s'occuper de différends entre les Etats et de toute situation qui constitue ou qui est susceptible de constituer une menace ou une rupture de la paix ou encore une agression. Le terme situation est à prendre tout aussi bien dans le sens des faits observés entre les Etats que ceux, à l'intérieur d'un Etat ou encore non étatique. Tel est, le cas de la situation en République Démocratique du Congo.

\section{Le Conseil économique et social}

Devenu organe principal sous l'impulsion de petits Etats, le Conseil Economique et Social comprend cinquante quatre membres dont le mandat a une durée de trois ans (Article 61 de la Charte).

Aux termes des articles 62 à 66 de la Charte, il est chargé de la coopération internationale dans les domaines prévus par les articles 1er, paragraphe 3 et 55 de la Charte.

\section{Le Conseil de tutelle}

En vertu de l'article 86 de la charte, il comprend trois catégories de membres. Toutefois, il faut noter que depuis la fin de la colonisation, sa fonction est tombée en désuétude.

\section{Le secrétariat}

Selon l'article 97 de la Charte, le Secrétariat comprend un Secrétaire Général à qui sont attachés plusieurs autres fonctionnaires internationaux et agents autant que peut exiger le besoin de l'organisation. Nommé pour un mandat de cinq ans renouvelable par l'Assemblée Générale sur recommandation du conseil de sécurité, l'article 97 in fine fait de lui le plus haut fonctionnaire de l'Organisation des nations Unies.

En fait, les fonctions du Secrétariat sont administratives et politiques. Les premières sont énoncées à l'Article 99 qui dispose : « Le Secrétariat Général peut attirer l'attention du Conseil de Sécurité sur toute affaire qui, à son avis, pourrait mettre en danger le maintien de la paix et de la Sécurité Internationales... »

\section{La Cour Internationale de Justice}

La Cour Internationale de Justice a pour mission de trancher les différends entre les Etats qui reconnaissent sa compétence. Tel a été le cas de l'Ouganda et de la République Démocratique du Congo.

Tout compte fait, l'Organisation des Nations Unies est une organisation internationale à vocation universelle. Fondée sur les décombres de la SDN, elle a pour mission primordiale le maintien de la paix et de la sécurité internationale dans le but d'assurer les meilleures 
conditions de vie aux peuples et le progrès social. Pour ce faire, elle est dotée des organes dont chacun remplit ses fonctions en synergie avec les autres. Le Conseil de sécurité ayant le monopole de la paix et de la sécurité internationales, peut intervenir, le cas échéant en vertu du chapitre VII, au moyen de la force par et à travers les moyens de la coercition militaire.

\section{B. L'ONU ET SES REGULATIONS POLITIQUES EN REPUBLIQUE DEMOCRATIQUE DU CONGO}

Dans ce dernier point, il est question de répondre à la problématique de la pertinence et de l'efficacité des régulations politiques onusiennes en faveur de la démocratie en République Démocratique du Congo. Pour ce faire, il va falloir d'une part, parler des différentes régulations politiques onusiennes dans ce pays, de leur pertinence et d'autre part à l'appréciation de leur efficacité et de l'attitude des dirigeants congolais vis-à-vis des interventions onusiennes avant de faire une lecture personnelle de la situation du Congo aujourd'hui malgré la présence de l'Organisation des Nations Unies.

\section{DE REGULATIONS POLITIQUES ONUSIENNES EN REPUBLIQUE DEMOCRATIQUE DU CONGO}

1. Intervention de l'Organisation des nations Unies dans les années soixante

L'histoire révèle que, l'implication des Nations Unies en faveur de la paix et de la sécurité en République Démocratique du Congo, ne date pas d'aujourd'hui. En effet, celle-ci était déjà intervenue dans les années soixante en République Démocratique du Congo.

A cette époque, on note l'immixtion des casques bleus dans la politique intérieure, contrôle de la station des radios, prise des armes pour le compte des troupes belges ou pour servir les ambitions d'un petit roitelet quelconque ${ }^{5}$.

Précisons à toute fin utile que, cette intervention aurait permis à la République Démocratique du Congo de reconquérir son unité.

\section{LES INTERVENTIONS RECENTES DE L'ONU EN REPUBLIQUE DEMOCRATIQUE DU CONGO}

Après ou même pendant les guerres qui ont déchiré la République Démocratique du Congo depuis la chute de la dictature mobutienne, les nations unies au travers les actions du Conseil de Sécurité sont intervenues plusieurs fois et continuent à intervenir dans ce pays, à travers plusieurs résolutions en vue, de la stabilisation de la paix et de l'instauration de la démocratie. De ces résolutions, il y a lieu de faire mention :

5 REILEUX, J.C., Mobutu : dignité pour l'Afrique, Paris, ed. Albani, état, 1988, p.53. 
- La résolution 1279 du Conseil de Sécurité en date du 30 novembre 1999 portant création de la MONUC. Selon Braeckmans; «La MONUC est une mission des Nations Unies en République Démocratique du Congo, pour continuer à la restauration de la paix et de la sécurité nationale suite aux conflits armés qui ont parsemé l'histoire politique du pays depuis le 2 août $1998 »^{6}$

- Après la résolution 1279 , le Conseil de Sécurité en a adopté cinq autres portant sur le conflit en République Démocratique du Congo au cours de l'année 2000. Il s'agit des résolutions 1291, 1304, 1316, 1326 et 1332.

- Au cours de l'année 2001, le même organe des Nations Unies a adopté trois résolutions relatives au conflit armé en République Démocratique du Congo.

C'est notamment les résolutions 1341,1355 et 1376.

- En 2002, trois résolutions toujours en rapport avec les conflits armés ont vu jour. Il s'agit des résolutions 1399, 1417 et 1445. En 2003, toujours dans le même contexte, le conseil de sécurité a adopté les résolutions 1457, 1468,1 484, 1489, 1493 et 1501.

- L'année 2004 sera marquée par l'adoption de 5 résolutions, à savoir les résolutions 1522 , $1533,1552,1555$ et 1565 .

- Au cours de l'année 2005 seront adoptées les résolutions 1592, 1612, 1616, 1621, 1635,1649 dans le but de résoudre les conflits armés congolais.

- L'année 2006 sera celle des résolutions 1654, 1665, 1671, 1692, 1711, 1736 toutes gardant en vue le mandat reconnu à la MONUC par la résolution 1565.

- En 2007, six autres résolutions seront adoptées, à savoir la résolution 1742, 1751, 1768, 1771, 1794.

- Durant l'année 2008, la résolution 1856 confie à la MONUC entre autres la protection des civils, du personnel humanitaire et des installations des Nations Unies, le désarmement et démobilisation des groupes armés étrangers et congolais et surveillance des moyens dont ils disposent: «Formation et accompagnement de FARDC à l'appui de réformes du secteur de la sécurité du territoire de la République Démocratique du Congo, la consolidation des institutions démocratiques et de l'Etat de droit. Le paragraphe 8 de cette résolution stipule : "qu'il importe que la MONUC s'acquitte intégralement du mandat énoncé dans la présente résolution ».

- En 2009, la résolution 1906 reprend le mandat assigné à la MONUC par la résolution 1856 en y précisant quatre priorités, à savoir assurer la protection des civils et le respect de leurs droits et statut conformément aux principes du droit humanitaire, des droits de l'homme et des réfugiés, éliminer la menace que représentent les groupes armés congolais et étrangers, etc.

- En 2010, la MONUC est remplacée par la MONUSCO sur base de la résolution 1925 du 28 mai 2010. son mandat est d'assurer la protection des civils, à la différence de la MONUC dont la mission était de faire respecter l'accord de Cessez-le-feu de Lusaka, d'ame-

6 BRAECKMAN, C, Les Bouveaux prédateurs, éd. Fayard, Paris, 2000, p.47. 
ner les congolais à un accord global et inclusif, de mettre sur pied un gouvernement d'union nationale, de réconciliation et transition, et de les accompagner aux élections libres, transparentes, démocratiques et inclusives à tous les niveaux.

- En 2011, l'année des élections à problèmes, L'Organisation des Nations Unies intervient par la MONUSCO dans les limites de la résolution 1991. La mission onusienne doit travailler avec le gouvernement Congolais pour améliorer les opérations militaires au Kivu et dans la Province Orientale; asseoir l'autorité de l'Etat, appuyer l'Organisation et la tenue des élections nationales, provinciales et locales, etc.

- De 2012 à 2015, multiples résolutions seront adoptées par le Conseil de sécurité, notamment les résolutions 2053, 2076,2078, 2098, 2136, 2147, 2198, 2211 dont la plus significative est la résolution 2098 (2013) portant sur la création de l'appui d'une brigade d'intervention de l'Organisation des Nations Unies aux forces armées et de sécurité.

- En 2016, soit le 30 mars, la résolution 2277 est prise suite à la persistance de la situation de menace de la paix et de la sécurité que représente encore la République Démocratique du Congo.

La dernière résolution est celle de 2017 donnant mandat à la MONUSCO de s'impliquer de manière directe et active dans l'organisation des élections.

Lors de la visite à Kinshasa après rencontre avec le Président de la République le 16 juin 2017, le Secrétaire Général adjoint chargé du maintien de la paix, se confiant aux antennes de la Radio Okapi a déclaré : « Le problème revient aux congolais eux-mêmes. C'est aux dirigeants congolais de prendre à cœur ce problème. Quant à nous, l'Organisation des Nations Unies joue le rôle d'accompagnement et souhaite l'organisation des élections en 2017 selon l'accord de la Saint Silvestre. "

Cependant, la question qui se pose à ce niveau d'analyse est celle de savoir quelles est la pertinence et l'efficacité de toutes ses régulations? Qu'en pensent les Congolais?

\section{DE LA PERTINENCE ET DE L'EFFICACITE DES REGULATIONS POLITIQUES ONUSIENNES EN REPUBLIQUE DEMOCRATIQUE DU CONGO}

Notons de prime abord que, la pertinence des régulations politiques onusiennes en République Démocratique du Congo reste plus une question liée à leur fondement juridique qu'à leur efficacité. C'est autant dire qu'il y a une distinction à faire entre la question de la qualité de l'ONU à s'impliquer dans le processus du maintien de la paix et de la sécurité en République Démocratique du Congo, donc de la démocratisation des institutions du pays et les résultats escomptés à travers ces régulations.

En fait, si l'on prend en compte la fait que la République Démocratique du Congo fait partie de l'Organisation des Nations Unies et les objectifs de cette dernière, il y a lieu de conclure à la pertinence de son implication dans la situation qui prévaut dans ce pays.

Ces implications ne sont pas une ingérence dans les affaires intérieures du Congo mais une action rentrant dans les prérogatives de l'Organisation des Nations Unies. En effet, à moins d'ignorer le pourquoi de la création de cette organisation, l'on peut considérer ses di- 
verses interventions dans la recherche d'une solution durable aux problèmes de la République Démocratique du Congo comme constituant une ingérence dans les affaires internes du pays ou une violation de sa souveraineté. L'on retiendra à toute fin utile que l'Organisation des Nations Unies n'est pas un Etat mais une Organisation Interétatique. En tant que telle, elle a des prérogatives qui ne peuvent, dans certains cas, être freinées dans leur exercice par le principe de la non-ingérence ou de la souveraineté car, la paix et la sécurité internationales se situent au-dessus de toute souveraineté.

S'agissant de l'efficacité de ses régulations, il y a lieu de dire que celles-ci sont efficaces. Il s'agit cependant d'une efficacité relative; car tout n'est pas encore complètement réalisé. Les élections ont eu lieu mais partiellement et actuellement la machine est bloquée. Les poches des forces négatives ont diminué en nombre et leurs opérations ne sont plus intensives. Il reste cependant à améliorer son action dont l'objectif idéal qu'est l'instauration de la démocratie et la décentralisation des congolais n'est pas encore atteint. Sachant que les congolais veulent partir sans se départir, l'efficacité de l'action onusienne ne sera effectivement acquise que par l'application des prérogatives reconnues au Conseil de Sécurité, celle de recourir, le cas échant, à la force pour démanteler la classe politique congolaise au pouvoir. Cette solution serait plus efficace et dissuasive car, la classe politique congolaise au pouvoir n'a plus une lecture rationnelle des événements et de l'histoire du Congo. Si elle est arrivée au pouvoir, c'est dans des circonstances tumultueuses avec tout ce qu'elles impliquent. Dès lors, ne pas organiser les élections, organiser des dialogues dont on sait d'avance qu'ils n'apporteront des solutions autres que celles que l'on voudrait, multiplier les tentatives de saper la Constitution même dans ses articles verrouillés, crier à l'ingérence des puissances étrangères ou de l'Organisation des Nations Unies dans des questions de souveraineté, tout cela est une preuve éloquente d'un refus manifeste de mettre en application, de bonne foi, les principes de l'Organisation des Nations Unies dont on est censé être membre. Pour plus d'efficacité dans ses régulations et ses actions, il ne reste plus à l'Organisation des Nations Unies que d'agir en fonction de l'Article VII de la Charte : "L'utilisation de la force pour faire partir du pouvoir une classe politique dont l'objectif est de mettre en péril la paix et la sécurité internationales à partir de la République démocratique du Congo. »

\section{CONCLUSION}

L'histoire politique de la République Démocratique du Congo nous a semblé être une histoire sui generis. Depuis son indépendance jusqu'à ce jour, ce pays reste toujours plongé dans une crise politique récurrente de contestation de légitimité des institutions et de leurs animateurs dont les conséquences ne semblent pas donner de leçon aux Congolais et à leurs dirigeants. Après des avancées significatives sur le plan de la paix et de la démocratie sous l'impulsion de l'Organisation des Nations Unies (ONU) depuis les guerres qui l'ont récemment déchirée, la République Démocratique du Congo est devenue de nos jours un champ de tripatouillages politiques aux conséquences désastreuses pour son développement et 
l'instauration d'un Etat démocratique et, donc de Droit, gage de la paix et de la sécurité tant au niveau national qu'international.

Aussi, avons-nous estimé, seul l'usage de la force contre la classe politique congolaise au pouvoir pourrait rendre plus efficaces les régulations onusiennes en faveur de la démocratie.

Ce mécanisme n'est donc pas une violation pour l'ONU de principes qui la régissent, mais plutôt une expression de son engagement en faveur de l'intérêt supérieur de l'humanité au Congo; la paix et la sécurité internationales étant continuellement menacées en République Démocratique du Congo à cause du comportement antidémocratique de sa classe politique au pouvoir.

\section{BIBLIOGRAPHIE}

\section{A. TEXTES LEGAUX}

La Constitution de la République Démocratique du Congo du 18 février 2006 telle que modifiée à ce jour par la loi n¹1-002 du 20 janvier 2011.

La Charte des Nations Unies du 1945, San Francisco.

\section{B. OUVRAGES}

BRAEKMAN, C., Les nouveaux prédateurs, Fayard, Paris, 2000.

Didier ROUGET, Le Guide de la Protection Internationale des droits de l'homme, Dalloz, Paris, 2000. KADORY, N.K., Organisation Internationale, éd. Essai, Lubumbashi, 2013.

REILLEUX, J.C., Mobutu : Dignité pour l'Afrique, Paris, éd. Albonm, état, 1988.

ZORGBRIDE, CH., Les Organisations Internationales, PUF, Paris, col. Que suis-je?

\section{WEBOGRAPHIE}

Wilkipédia : «Programme Alimentaire Mondial»

Wilkipédia : Programme des Nations Unies pour le Développement.

Wilkipédia, Organisation Mondiale de la Santé.

Wilkipédia : Groupe de la Banque Mondiale. 